\title{
Constrained control of arrays of wave energy devices
}

\section{Giorgio Bacelli*, John Ringwood}

Center for Ocean Energy Research, NUI Maynooth, Maynooth, Co. Kildare, Ireland

\section{A R T I C L E I N F O}

\section{Keywords:}

Array

Control

Global

Independent

Constraints

\begin{abstract}
A B S T R A C T
It is well known in the literature that the total absorbed energy of a farm of wave energy converter depends, within a wide range of variability, on the hydrodynamic interaction between the individual devices. Several studies have addressed the problem of controlling the wave energy converters so as to exploit the positive interaction between the devices in order to increase the total amount of absorbed energy.

This paper studies two types of control systems for array of wave energy converters: the first is similar to the classical optimal control which uses the complete (linear) hydrodynamic model of the array and information about the status (velocity) of the other devices, while the second is a suboptimal control systems which calculates the optimal motion while neglecting completely the interaction between the devices. Most importantly the comparison is carried out considering the effects of constraints on the maximum allowed oscillation amplitude and PTO force.
\end{abstract}

(c) 2013 Elsevier Ltd. All rights reserved.

\section{Introduction}

Wave energy converters (WECs) are likely to be deployed in arrays composed of multiple devices, in order to both exploit the eventual economy of scale and to exploit the positive hydrodynamic interaction. The design of such a positively-interacting collective depends on a number of factors, including the wave farm layout. In addition, the optimality of the layout (spacing, orientation, etc), with respect to positive reinforcement, depends on sea state and incident wave direction, which are variable.

\footnotetext{
* Corresponding author.

E-mail addresses: giorgio.bacelli@eeng.nuim.ie (G. Bacelli), john.ringwood@eeng.nuim.ie (J. Ringwood).
} 
For a given wave farm layout, however, the motion of each device determines the radiated wave patterns, which are a significant function of the control strategy employed on each device. This opens the possibility that devices can, via inter-device communication, coordinate their motion to the mutual benefit of all devices in the farm, with maximum farm power production as an objective. Such a structure, for a simple 2-device array, is shown in Fig. 1.

Wave energy arrays include both large devices (circa $1 \mathrm{MW}$ ), individually moored, and closelypacked arrays of devices, which are contained within a larger superstructure. Examples of larger individual devices include Pelamis [1], Oyster [2] and Powerbuoy [3], while closely-spaced arrays include those by Wavestar [4], Fred Olsen Lifesaver [5] , Manchester bobber [6] and Trident Technologies [7]. In particular, compact circular arrays have been shown to be capable of extracting more energy than a single device of the same volume, and presenting a larger spectral bandwidth [8].

Original work in the area of global control of arrays was carried out by Evans [9] and Falnes [10], who developed optimal control for a number of oscillating bodies. Subsequently, Justino and Clement [11] developed a suboptimal control strategy, followed in a similar approach by other works as in $[12,13,7]$. Linear damping has also been widely considered for the control of array and it is generally divided into two categories: the first is where all the devices have the same damping coefficient, such as in[14-17], while the second is where the damping of each device composing the array has been selected by some optimisation criteria so as to maximise a given objective, which is generally the total absorbed energy, as in [13-15].

In this paper, two control strategies are compared for the control of WEC arrays, namely Global Control (GC) and Independent Control (IC). Both GC and IC are model-based control strategies; however, GC is based on a centralised control algorithm which uses the complete hydrodynamic model of the array whereas, with IC, each device is controlled independently using the hydrodynamic model of a single isolated device. For comparison purposes, it is assumed that the total local hydrodynamic force on each WEC can be estimated for both GC and IC, where the total hydrodynamic force is intended to be the sum of the force exerted by the incoming wave, diffraction and radiation. While we consider several layouts, separations and body geometries, it is not the objective of this study to conclude on optimal shapes or layouts, but rather to achieve some consistency of conclusion. However, it is appreciated that WEC array performance is sensitive to both layout and device geometry [18]. The comparison if the two control strategies is carried out also with respect to constraints on the PTO and oscillation amplitude. Original work in the study of constrained control of system of oscillating bodies was carried out by Evans [19] and Thomas and Evans [18], followed by Falnes and Budal [20]. Also Fitzgerald and Thomas [21] considered array of devices with oscillation amplitude constraints, as well as DeBacker et al. [14]. However, constraints on the PTO force have only been considered for the single device case as in Hals et al. [22] and Cretel et al. [23].

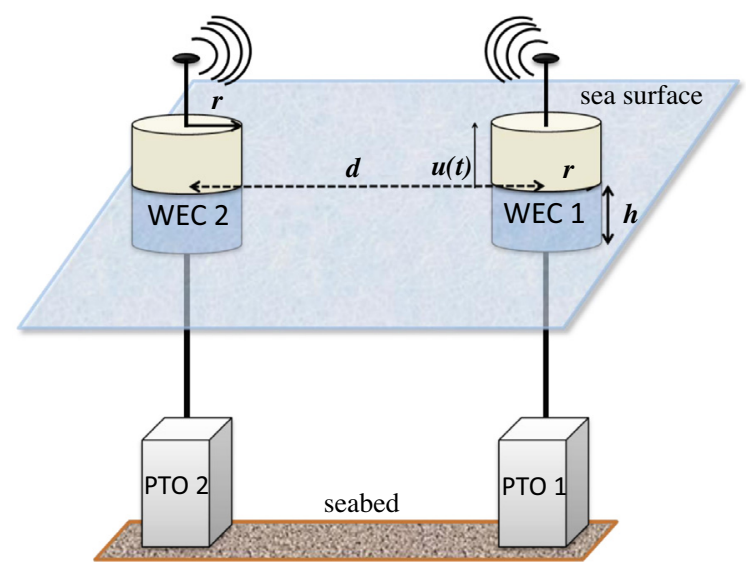

Fig. 1. Layout for a 2-body array. Motion and PTO force is restricted to heave only. 
We formulate the optimal GC and IC problems in the time domain, which are then discretised and solved as nonlinear programs. A preliminary analysis of the constrained GC problem using this approach was already reported in [24]. In the literature, the predominant metric used to evaluate the performance of a WEC array is the q-factor [18], which indicated the average device performance of an array element compared to an isolated device. In this paper, however, we consider the ratio of the total array (energy) performance for the GC and IC cases, because the main objective is to compare the performance of these two control strategies for any given WEC in any given array configuration. The q-factor allows the evaluation of the absorption performance for an ensemble of devices with respect to an isolated device, and it provides a useful metric for the comparison of the overall performance of different array configurations. However, the q-factor does not allow to discern between the effect of the control strategy and the hydrodynamics on the total absorbed power, that is, two control strategies may provide different total absorbed power while having the same q-factor.

\section{Formulation of hydrodynamic model}

The control systems are implemented on different array layouts and for several geometries of the WECs composing the arrays. The WECs are vertical cylinders of radius $r$, draught $h$ and the distance between their vertical axis is denoted with $d$, as depicted in Fig. 1 for the particular case of a two-body array.

\subsection{Equations of motion}

For this work we assume linear wave theory and non-compressible irrotational flow. Therefore, the motion of the device can be described, in the time domain, by Cummin's equation [25]:

$$
\mathrm{M}_{t} \ddot{u}(t)+\mathrm{B} \dot{u}(t)+\int_{t_{0}}^{t} \mathrm{~K}(t-\tau) \dot{u}(\tau) d \tau+\mathrm{S} u(t)=f(t),
$$

where $u(t) \in \mathbb{R}^{m}$ is the vector of the vertical positions of the WECs, and $m$ the number of degrees of freedom of the system, which corresponds to the number of WECs composing the array, because the motion of each device is assumed to be restricted to heave only. $\mathrm{M}_{t}=\mathrm{M}+\mathrm{m}_{\infty}$ where $\mathrm{M} \in \mathbb{R}^{m \times m}$ is the generalised mass matrix and $\mathrm{m}_{\infty} \in \mathbb{R}^{m \times m}$ is the asymptotic values of the added mass at infinite frequency; $\mathrm{B} \in \mathbb{R}^{m \times m}$ is the viscous damping term; $\mathrm{S} \in \mathbb{R}^{m \times m}$ is the hydrodynamic stiffness and $\mathrm{K}(t) \in \mathbb{R}^{m \times m}$ is the matrix of the radiation impulse responses. The vector of external forces $f(t) \in \mathbb{R}^{m}$ is given by $f(t)=f_{e}(t)+f_{p t o}(t)$ where $f_{e}(t)$ is the exciting force and $f_{\text {pto }}(t)$ is the PTO force. The excitation force is calculated as $f_{e}(t)=\mathcal{F}^{-1}\{\mathrm{X}(\omega) \eta(\omega)\}$, where $\eta(\omega)$ is the Fourier transform of the wave elevation and $X(\omega)$ is the frequency domain exciting force transfer function.

\subsection{Numerical modelling}

The hydrodynamic coefficients $\mathrm{m}_{\infty}$ and $\mathrm{K}(t)$ are calculated from the frequency domain radiation impedance matrix $Z(\omega)$ by applying Ogilvie's relations [26,27]. The matrices $X(\omega), Z(\omega)$ and $S$ are then computed in the boundary element solver WAMIT ${ }^{\circledR}[28]$. This package is widely applied in studies of WEC arrays, as in $[14,16,17]$. In WAMIT the high order method is used and computations are performed for 160 frequencies equally spaced at intervals of $0.0151 \mathrm{rad} / \mathrm{s}$. All control simulations are implemented in MATLAB ${ }^{\circledR}$ and are performed with the same frequency resolution as the WAMIT output. The software simulates the motion of the devices, the PTO forces, the instantaneous converted power and the vertical velocities and displacements of the WECs for a representative surface elevation time-history of a given wave spectrum.

\subsection{Viscous damping approximation}

The viscous effect of a fluid on a body is usually described, under certain conditions [29], as a force proportional to the square of the relative velocity between the body and the fluid surrounding the 
body; that is $f_{d}=(1 / 2) C_{d} \rho \dot{u}|\dot{u}| A$, where $\rho$ is the fluid density, $A$ is the area of the body projected onto the plane orthogonal to the velocity $\dot{u}$, and $C_{d}$ is the drag coefficient, which is obtained experimentally (see for example Appendix 2 in [29]). Several recent studies have considered the effects of viscosity on WEC motion, such as $[7,30,31]$.

The objective of the approximation is to find a force $\hat{f}_{d}$ which is linearly proportional to the velocity and that dissipates the same amount of energy as the force $f_{d}$. This procedure is known as Lorentz linearisation [32], and it has been used in the case of WECs by Folley et al. [33]. The approximation is carried out by equating the work of the nonlinear force $f_{d}$ with the work of the linear approximation $\hat{f}_{d}=B_{v} \dot{u}$ which result in $\int_{0}^{T} f_{d} \dot{u} d t=\int_{0}^{T} \hat{f}_{d} \dot{u} d t$. By means of simple manipulations, the damping coefficient $B_{v}$ can be expressed as

$$
B_{v}=\frac{C_{d} \rho A \int_{0}^{T} \dot{u}^{2}(t)|\dot{u}(t)| d t}{2 \int_{0}^{T} \dot{u}^{2}(t) d t} .
$$

For the special case of a vertical cylinder of radius $r$ oscillating sinusoidally in heave with velocity $\dot{u}(t)=U \cos (\omega t)$, the coefficient $B_{v}$ is:

$$
B_{v}=4 C_{d} \rho r^{2} \omega U / 3 \text {. }
$$

Knowledge of the velocity is required to calculate the coefficient $B_{v}$, but the velocity itself depends on $B_{v}$, therefore an iterative procedure has been implemented. The procedure is initiated by setting $B_{v}$ to an initial value $B_{v}^{0}$, which is not critical for the convergence because the relation between $B_{v}$ and the velocity $\dot{u}$ is monotonic ( $\dot{u}$ decreases when $B_{v}$ increases). The $i$-th iteration of the procedure is composed of two steps, which are:

(1) Calculate the velocity $\dot{u}^{i}$ using the value of $B_{v}^{i-1}$

(2) Calculate $B_{v}^{i}$ using the velocity $\dot{u}^{i}$ and the formula (2), or (3) in the case of regular waves.

The procedure is stopped when the difference between two subsequent values of $B_{v}$ is smaller than a threshold $\delta$, that is $\left|B_{v}^{i}-B_{v}^{i-1}\right|<\delta$. For any given geometry, controller type and sea state, the coefficient $B_{v}$ is calculated by simulating an isolated device.

\section{Energy maximising control}

The control problem is defined as it follows: find the optimal profile for the PTO forces which maximises the total energy absorbed by the array described by the equation of motion (1) over a time interval of length $T$. The total energy absorbed by the array is considered to be the sum of the mechanical work performed by each of the PTO forces as:

$$
W=-\sum_{k=1}^{n} \int_{0}^{T} \dot{u}^{k}(t) f_{p t o}^{k}(t) d t
$$

where $\dot{u}^{k}(t)$ and $f_{p t o}^{k}(t)$ are, respectively, the heave velocity and the PTO force of the $k$-th device.

In the ideal case, assuming the wave excitation is known completely into the future, the optimisation is performed over an infinitely long the time interval, that is for $T \rightarrow+\infty$; however, it has been shown [34] that a limited horizon suffices to achieve close to optimal energy absorption. As a consequence, the real-time implementation of the control algorithm can be performed in a receding horizon fashion, as described in [35], where future knowledge of either wave elevation or excitation force, up to time $T$ ahead, is obtained by prediction. Since the focus of this paper is on the control of arrays of WECs, we neglect the effects of prediction by applying the separation principle [36], which is a standard approach in control engineering, allowing the design of the optimal control to be separated from the design of the predictor; thus perfect knowledge of future wave elevation is assumed, in order to isolate the effects of control on the total energy produced. 


\subsection{Discretisation}

The control problem is discretised by approximating the velocity and the PTO force with a linear combination of basis functions, resulting in a finite dimension optimisation problem. In this paper, trigonometric functions are chosen as basis functions, thus the PTO force and the velocity are approximated with the truncated zero-mean Fourier series

$$
\begin{aligned}
& \dot{u}_{k}(t) \approx \sum_{n=1}^{N / 2} v_{k}^{n, c} \cos \left(n \omega_{0} t\right)+v_{k}^{n, s} \sin \left(n \omega_{0} t\right), \\
& f_{p t o_{k}}(t) \approx \sum_{n=1}^{N / 2} \phi_{k}^{n, c} \cos \left(n \omega_{0} t\right)+\phi_{k}^{n, s} \sin \left(n \omega_{0} t\right) .
\end{aligned}
$$

The best approximation of the solution for the equation of motion (1) is sought by applying the Galerkin method, the details of which are presented in [35] , and the result is the linear system

$$
\mathrm{GX}=P+\mathrm{E},
$$

where $X, P, \mathrm{E}$ and $\mathrm{G}$ are defined as

$$
X=\left[\begin{array}{l}
X_{1} \\
X_{2}
\end{array}\right] \quad P=\left[\begin{array}{l}
P_{1} \\
P_{2}
\end{array}\right] \quad E=\left[\begin{array}{l}
E_{1} \\
E_{2}
\end{array}\right] \quad G=\left[\begin{array}{ll}
G_{11} & G_{12} \\
G_{21} & G_{22}
\end{array}\right] .
$$

The vectors $X_{k}$ and $P_{k}$, for $k=1,2$, are the vectors of the Fourier coefficients of the velocity and PTO force of the $k$-th device, respectively, and are arranged as

$$
\begin{aligned}
& X_{k}=\left[v_{k}^{1, c}, v_{k}^{1, s}, v_{k}^{2, c}, v_{k}^{2, s}, \ldots, v_{k}^{\frac{N}{2}, c}, v_{k}^{\frac{N}{2}, s}\right]^{T} \\
& P_{k}=\left[\phi_{k}^{1, c}, \phi_{k}^{1, s}, \phi_{k}^{2, c}, \phi_{k}^{2, s}, \ldots, \phi_{k}^{\frac{N}{2}, c}, \phi_{k}^{\frac{N}{2}, s}\right]^{T}
\end{aligned}
$$

The elements of the vectors $\mathrm{E}_{k}$ are the Fourier coefficients of the excitation force on the $k$-th device and are arranged in the same manner as the vectors $X_{k}$ and $P_{k}$. The matrices $G_{i j} \in \mathbb{R}^{N \times N}$ composing the matrix $\mathrm{G}$ are block diagonal, where each of the $N / 2$ square blocks is of size two and the $l$-th block is defined as

$$
\begin{aligned}
& \mathrm{G}_{i j}^{l}=\left[\begin{array}{cc}
\mathrm{D}_{i j}^{l} & \mathrm{M}_{i j}^{l} \\
-\mathrm{M}_{i j}^{l} & \mathrm{D}_{i j}^{l}
\end{array}\right] \quad \text { for } l=1, \ldots, N / 2 \\
& \mathrm{D}_{i j}^{l}=\mathrm{R}_{i j}\left(l \omega_{0}\right)+\mathrm{B}_{i j} \\
& \mathrm{M}_{i j}^{l}=l \omega_{0}\left(\mathrm{M}_{i j}+\mathrm{m}_{i j}\left(l \omega_{0}\right)\right)-\mathrm{S}_{i j} /\left(l \omega_{0}\right) .
\end{aligned}
$$

$\mathrm{B}_{i j}, \mathrm{M}_{i j}$ and $\mathrm{S}_{i j}$ are, respectively, the elements of the matrices $\mathrm{B}, \mathrm{M}$ and $\mathrm{S}$, while $\mathrm{R}_{i j}(\omega)$ and $\mathrm{m}_{i j}(\omega)$ are the elements of the radiation impedance matrix $Z(\omega)$, which is computed by WAMIT and is defined as $\mathrm{Z}(\omega)=\mathrm{R}(\omega)+i \omega \mathrm{m}(\omega)$.

\subsection{Constraints}

Physical limitations of the WECs are taken into account by introducing constraints on their motion. In particular, an oscillation amplitude constraint is introduced to reflect the finite excursion of the PTO, and an anti-slamming constraint to prevent the device from completely emerging from, and being submerged by, water.

\subsubsection{Oscillation amplitude constraints}

The oscillation amplitude constraint for the $k$-th device is defined as $\left|u_{k}(t)\right| \leqslant U_{k}$. The approximated position is obtained by integrating (5) and the resulting amplitude constraint is: 


$$
\left|\sum_{n=1}^{N / 2} x_{k}^{n, c} \frac{\sin \left(n \omega_{0}\right)}{n \omega_{0}}-x_{k}^{n, s} \frac{\cos \left(n \omega_{0}\right)}{n \omega_{0}}\right| \leqslant \mathrm{U}_{k},
$$

where the condition $\sum x_{k}^{n, s}=0$ is imposed to obtain a zero-mean position. Since it is not possible to find the maximum of a Fourier series analytically, the constraint is imposed only at specified time instants $t_{\mu}$ in the interval $[0, T]$, defined as $t_{\mu}=\mu \Delta t$ for $\mu=0,1, \ldots, N_{c}$, and where $T=N_{c} \Delta t$. Consequently, the approximated amplitude constraints result in the $4 N_{c}$ linear inequalities in the vector $P$

$$
\begin{aligned}
& \mathrm{S}_{\mu} \mathrm{G}^{-1} P \leqslant \mathrm{U}-\mathrm{S}_{\mu} \mathrm{G}^{-1} \mathrm{E} \\
& -\mathrm{S}_{\mu} \mathrm{G}^{-1} \mathrm{P} \leqslant \mathrm{U}+\mathrm{S}_{\mu} \mathrm{G}^{-1} \mathrm{E} \quad \text { for } \mu=0, \ldots, N_{c}
\end{aligned}
$$

where $U=\left[U_{1}, U_{2}\right]^{T}$ and the matrices $S_{\mu} \in \mathbb{R}^{2 \times 2 N}$ are constructed from the matrix $S$ and the vectors $T_{\mu}$ as in (11). The matrix $S \in \mathbb{R}^{N \times N}$ is block diagonal with a total of $N / 2$ square blocks of size 2 , where the $l$-th block $\mathrm{S}_{l}$ is defined as

$$
\mathrm{S}_{l}=\left[\begin{array}{cc}
0 & \frac{1}{l \omega_{0}} \\
-\frac{1}{l \omega_{0}} & 0
\end{array}\right], \quad \mathrm{S}_{\mu}=\left[\begin{array}{cc}
\mathrm{ST}_{\mu} & 0_{1 \times N} \\
0_{1 \times N} & \mathrm{ST}_{\mu}
\end{array}\right] .
$$

The vector $\mathrm{T}_{\mu} \in \mathbb{R}^{N}$ is defined as $\mathrm{T}_{\mu}=\mathrm{T}\left(t_{\mu}\right)$ where

$$
\mathrm{T}(t)=\left[\cos \left(\omega_{0} t\right), \sin \left(\omega_{0} t\right), \ldots, \cos \left(\frac{N \omega_{0} t}{2}\right), \sin \left(\frac{N \omega_{0} t}{2}\right)\right] .
$$

\subsection{PTO force constraint}

Similarly to the oscillation amplitude constraint, the PTO force constraint for the $k$-th device is defined as $\mid f_{p_{t o}}(t) \leqslant \mathrm{F}_{k}$. By noting that the approximated PTO force in (6), for $t=t_{\mu}$ can be written as

$$
f_{p t o_{k}}\left(t_{\mu}\right) \approx \mathrm{T}_{\mu} P_{k}
$$

where $P_{k}$ are the components of the PTO force vector $P$ relative to the $k$-th device, the constraint on the PTO force can be expressed as the system of linear inequalities:

$$
\begin{aligned}
& {\left[\begin{array}{cc}
\mathrm{T}_{\mu} & 0_{1 \times N} \\
0_{1 \times N} & \mathrm{~T}_{\mu}
\end{array}\right] \quad P \leqslant \mathrm{~F}} \\
& {\left[\begin{array}{cc}
\mathrm{T}_{\mu} & 0_{1 \times N} \\
0_{1 \times N} & \mathrm{~T}_{\mu}
\end{array}\right] \quad P \leqslant-\mathrm{F} \quad \text { for } \mu=0, \ldots, N_{c}}
\end{aligned}
$$

\subsection{Global control}

The control system of the GC strategy is aware of the whole configuration of the array; the resulting optimisation problem is defined by the cost function $\mathrm{W}=-(T / 2) P^{T} X$, which is obtained by substituting (5) and (6) into the definition of the total absorbed energy in (4). If $G$ is non-singular, the cost function can be expressed as a function of $P$ by solving (7) w.r.t $X$, and the coefficients $P^{\star \varkappa}$ of the optimal PTO forces that maximise the absorbed energy for the array are obtained by solving the optimisation problem $^{1}$

$$
P^{\star}=\arg \max _{P}-P^{T} \mathrm{G}^{-1} P-P^{T} \mathrm{G}^{-1} \mathrm{E},
$$

subject to the linear inequality constraints in (10) or (12).

\footnotetext{
${ }^{1}$ The term $T / 2$ is a positive scaling factor that can be neglected for the calculation of the optimal PTO force because the vector $P^{\text {th }}$ which maximises $\mathrm{W}=-(T / 2) P^{T} X$ also maximises the cost function $\bar{W}=-P^{T} X$.
} 


\subsection{Independent control}

For the IC case, it is assumed that each device is equipped with its own controller and an excitation force estimator. It is also assumed that no communication occurs between the devices, and each controller uses the model of a single isolated device. That is, the control system of each device uses the model (1) where the variables $u(t)$ and $f(t)$ are scalars and where $\mathrm{M}_{t}, \mathrm{~B}, \mathrm{~K}(t), \mathrm{S}$ are also scalars and they are the parameters of a single isolated device with the same shape and dimensions of the devices composing the corresponding array. For the example case of an array composed of two WECs (as shown in Fig. 1, the controllers of devices 1 and 2 use, respectively, the discretised models

$$
\begin{aligned}
& \mathrm{G}_{s} X_{1}=P_{1}+\overline{\mathrm{E}}_{1} \\
& \mathrm{G}_{s} X_{2}=P_{2}+\overline{\mathrm{E}}_{2}
\end{aligned}
$$

where $G_{s} \in \mathbb{R}^{N \times N}$ is analogous to the matrix $G$ in the approximated equation of motion of the array in (7). However, in this case, $G_{s}$ is calculated using the hydrodynamic coefficients of a single isolated device. In particular, $G_{s}$ is a block diagonal matrix with square blocks of size two, and is constructed in the same manner as the diagonal blocks $\mathrm{G}_{i i}$ of the matrix $\mathrm{G}$, as described in (9) for $i=j$, with the difference being that the elements $D_{i j}^{l}$ and $M_{i j}^{l}$ of $G_{s}$ are calculated using the radiation impedance matrix of a single isolated device.

$\overline{\mathrm{E}}_{k}$ is the excitation force measured by the estimator on WEC $k$; more specifically, $\overline{\mathrm{E}}_{1}=\mathrm{E}_{1}-\mathrm{G}_{12} X_{2}$ and $\bar{E}_{2}=E_{2}-G_{21} X_{1}$. In fact, it is assumed that the excitation force estimator on each device is not capable of discerning the excitation force due to incoming waves from the radiation generated by other bodies; therefore, the estimator provides a signal which is the sum of the radiation force caused by other bodies $\left(G_{12} X_{2}\right.$ and $\left.G_{21} X_{1}\right)$ and excitation from incoming waves $\left(E_{1}\right.$ and $\left.E_{2}\right)$.

Each of the independent controllers calculates the optimal PTO force that maximises the energy absorbed by the corresponding WEC using the models in (14) and (15). The optimal PTO forces ( $P_{1}^{*}$ and $P_{2}^{*}$ ) are the solutions of the optimisation problems

$$
\begin{aligned}
& P_{1}^{*}=\arg \max _{P_{1}} W_{1}=\arg \max _{P_{1}}-P_{1}^{T} \mathrm{G}_{s}^{-1} P_{1}-P_{1}^{T} \mathrm{G}_{s}^{-1} \bar{E}_{1} \\
& P_{2}^{*}=\arg \max _{P_{2}} W_{2}=\arg \max _{P_{2}}-P_{2}^{T} \mathrm{G}_{s}^{-1} P_{2}-P_{2}^{T} \mathrm{G}_{s}^{-1} \bar{E}_{2}
\end{aligned}
$$

the cost functions of which are the energy absorbed by each device, that is $W_{1}=-P_{1}^{T} X_{1}$ and $W_{2}=-P_{2}^{T} X_{2}$. However, the solutions of the optimisation problems in (16) and (17) are coupled because $P_{1}^{*}$ depends on $\bar{E}_{1}$, which is function of the velocity of body $2\left(X_{2}\right)$, and vice versa; the problem is then effectively solved iteratively. The initial condition is considered to be with the PTOs switched off $\left(P_{1}=P_{2}=\mathbf{0}\right)$. The velocities $X_{1}$ and $X_{2}$ are then calculated by means of the equation of motion (7) and the controller calculates the PTO forces by solving the optimisation problems (16) and (17). When the PTO forces are applied to the WECs, the new velocities are calculated again, using (7), and the process is repeated. The computations performed by the controller at the $n$-th step of the iteration are :

$$
\begin{aligned}
& X^{n}=G^{-1}\left(\widehat{P}^{n-1}+E\right) \\
& \widehat{P}_{1}^{n}=\arg \max _{P_{1}}-P_{1}^{T} \mathrm{G}_{s}^{-1} P_{1}-P_{1}^{T} \mathrm{G}_{s}^{-1}\left(E_{1}-\mathrm{G}_{12} X_{2}^{n}\right) \\
& \widehat{P}_{2}^{n}=\arg \max _{P_{2}}-P_{2}^{T} \mathrm{G}_{s}^{-1} P_{2}-P_{2}^{T} \mathrm{G}_{s}^{-1}\left(E_{2}-\mathrm{G}_{21} X_{1}^{n}\right),
\end{aligned}
$$

where $n>1, \widehat{P}^{0}=\mathbf{0}$ and $\widehat{P}^{n}, \widehat{P}_{1}^{n}, \widehat{P}_{2}^{n}, X^{n}, X_{1}^{n}, X_{2}^{n}$ are defined according to (8). The iteration stops when the PTO forces approach to their asymptotic values, which is implemented by the condition $\left\|P_{k}^{n}-P_{k}^{n-1}\right\|<\varepsilon \forall k=1,2$.

\subsubsection{Adaptive constraints for independent control}

The control models in (14) and (15) used by the ICs are not accurate because they neglect part of the hydrodynamic interaction between the WECs. Consequently, the solution of the ICs constrained optimal control problem given by (16) and (17) subject to the linear constraints (10) and/or (12) may produce a motion that violates one of the amplitude constraints. The constrained optimal control 
problem for the IC is the approached by first solving the optimal control problems defined in (16) and (17), with constraints (10) and (12). If the motion of any of the devices violates a constraint, the optimal control problem is solved again, but with a more restrictive constraint. In practice, if the motion of the $k$-th device violates its amplitude constraint $\mathrm{U}_{k}$, the control problem is solved again, but the new amplitude constraint $\bar{U}_{k}$ is $\bar{U}_{k}=\alpha \mathrm{U}_{k}$ with $\alpha<1$. The sequence is then repeated until all constraints are satisfied.

\subsection{Calculation of the solution of optimisation problem}

The optimisation problems for GC and IC are convex quadratic programs. In fact, it can be verified that the matrices $G$ and $G_{s}$ of the quadratic cost functions (13), (16) and (17) are positive definite and constraints (10) and (12) are linear. The active set algorithm [37] is used to solve the optimisation problems, which is implemented in Matlab by the function quadprog.

\section{Results}

We consider three possible array layouts (Fig. 2): layout 1 is composed of two heaving cylinders; layout 2 is a linear array of three WECs, while layout 3 is composed of three WECs placed at the vertices of an equilateral triangle. For each layout, we have chosen 24 values of the inter-body spacing $d$, logarithmically spaced between $2.2 r$ to $10 r$. Three device geometries for the WECs composing each array have been simulated, each of which has approximately the same volume $\left(\approx 160 \pi \mathrm{m}^{3}\right)$ but different resonant periods; Table 1 lists the radii $r$, draughts $h$ and resonant period $T_{r}$ for each device.

The optimal control laws for each WEC in the case of IC are obtained by iteration, and it is assumed that, between consecutive iterations, there is enough time for the waves radiated from a device to reach all the other devices, and enough time for the estimator and predictor on each WEC to build a reliable forecast of the incoming waves (reach quasi steady state). While this is not feasible in practice, it provides a best-case scenario for the IC case, providing an upper performance bound for the IC case. However, the comparison between the GC and the ICs is also carried out by considering only the first iteration of the ICs, which start from an initial condition where each PTO is switched off. The comparison of the GC with first iteration of the ICs is interesting from a practical point of view, since it highlights the effect of the PTO (and therefore the control system) on the interaction between devices.

Each of the three array layouts depicted in Fig. 2 has been simulated for all the three WEC geometries in Table 1. Both GC and ICs have been computed for each of the resulting nine possible arrays with inter-body spacing ranging between $2.2 r$ and $10 r$, and considering four Bretschneider spectra with $H_{\mathrm{s}}=1 \mathrm{~m}$ and $T_{p}=\{6,8,10,12\} \mathrm{s}$. The parameters $\omega_{0}$ and $N$ for the discretisation of the control problem are $N=160$ and $\omega_{0}=2 \pi / 200 \mathrm{rad} / \mathrm{s}$ respectively, while the thresholds $\delta$ and $\beta$ for the adaptive approximation of the viscous damping (Section 2.3) and the ICs (Section 3.5), respectively, are $\delta=1$ and $\varepsilon=10$. The simulations of both GC and IC have been carried out using the same model given by linear the equation of motion of the array in (1).

The patterns exhibited by the results are illustrated by the representative cases depicted in Figs. 3-5, which show the ratio between the energy absorbed using IC and GC $\left(E_{i g}=E_{i} / E_{g}\right)$, with respect to the normalized amplitude constraint $\bar{U}$ and normalizes force constraint $\bar{F}$, defined as

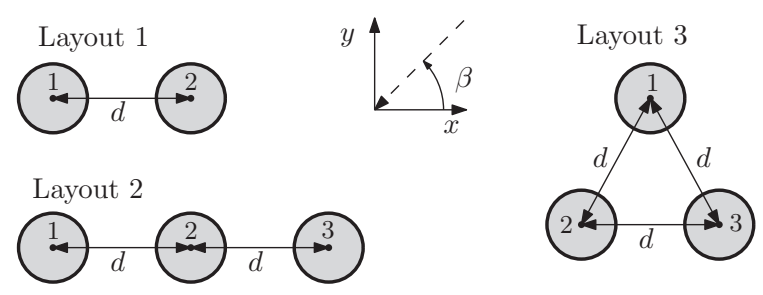

Fig. 2. Top view of array layouts and of incident waves angle $\beta$. 
Table 1

WECs dimensions and resonance period.

\begin{tabular}{llll}
\hline WEC geometry & 1 & 2 & 3 \\
\hline Radius $r(m)$ & 4 & 5 & 6.25 \\
Draught $h(m)$ & 10 & 6 & 4 \\
Resonance $T_{r}(s)$ & 7.1 & 5.9 & 5.4 \\
\hline
\end{tabular}
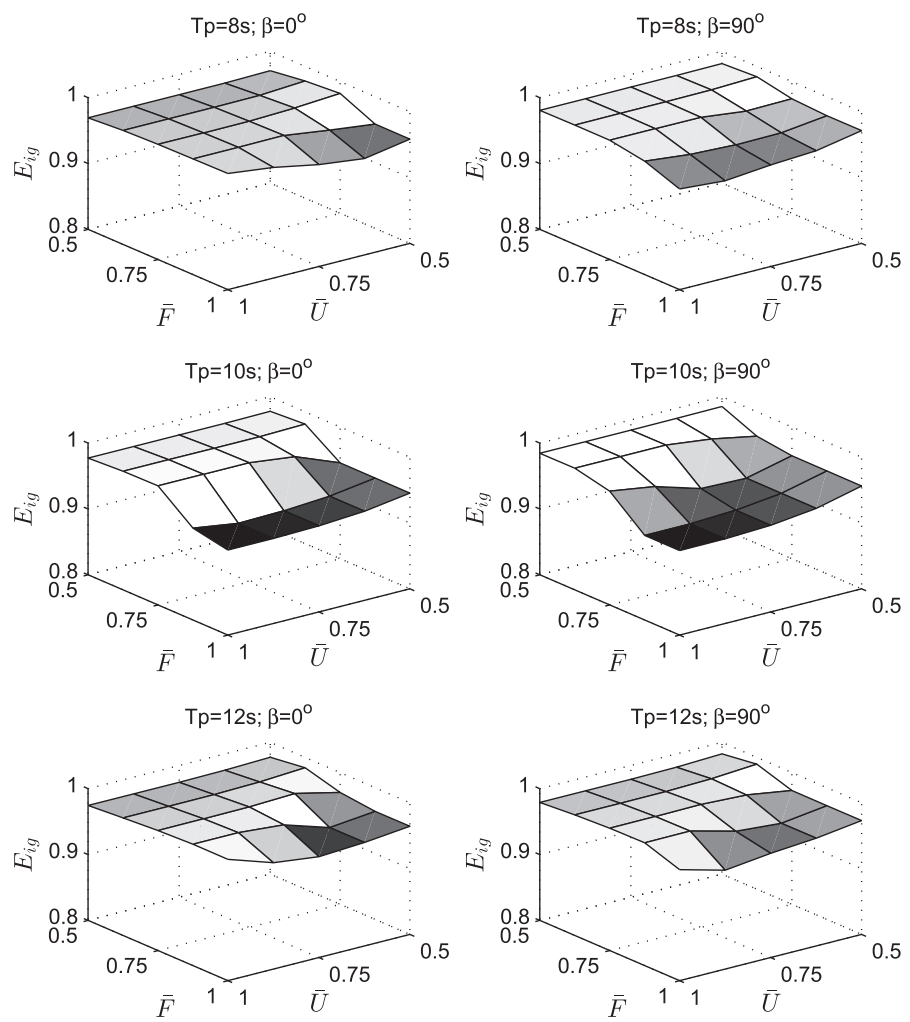

Fig. 3. Layout 1 ; geometry $2 ; d=20 \mathrm{~m}$.

$$
\bar{U}=\frac{U}{\max u_{u}^{1}} \quad \bar{F}=\frac{F}{\max f_{p t t_{u}}^{1}},
$$

where $u_{u}^{1}$ is the oscillation amplitude of a single isolated and unconstrained device, and $f_{\text {pto }}^{1}$ is the optimal PTO force of a single isolated and unconstrained device.

It can be noticed immediately that the relative performance of IC with respect of GC is more sensitive to the force constraint than the oscillation amplitude constraint. In particular, when restricting the maximum allowed PTO force, the performance of the IC approaches the performance of the GC $\left(E_{i g} \rightarrow 1\right)$. On the other hand, the relative performance of the IC degrades when imposing a more restrictive the oscillation amplitude constraint (i.e. when decreasing $\bar{U}$ ). These observations about the behaviour of the ratio $E_{i g}$ with respect to $\bar{U}$ and $\bar{F}$ are consistent for the three layouts and across several sea states and wave heading angles, as it can be seen by comparing Figs. 3-5.

Tables 2 and 3 provide a wider range of results for $E_{i g}$ as function of the peak period and the wave heading angle, for the array layouts 2 and 3, respectively. For each $T_{p}$ and $\beta$ the tables list three entries: 

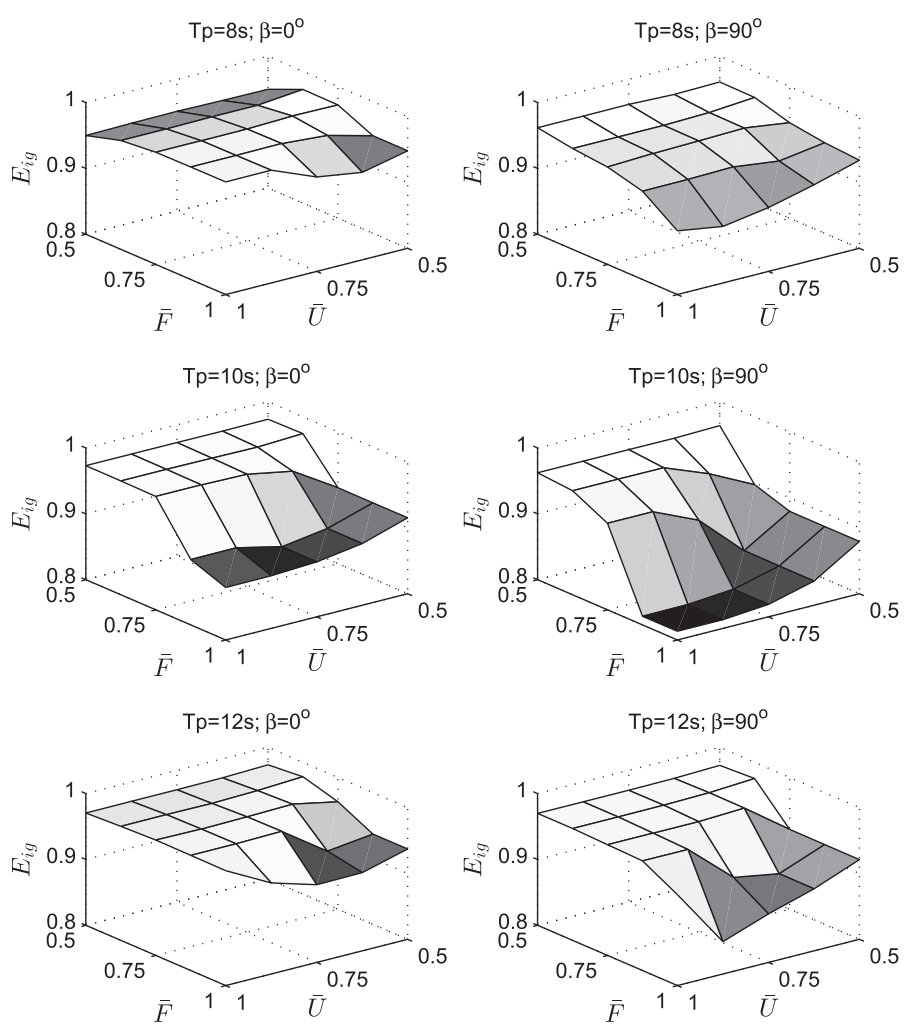

Fig. 4. Layout 2; geometry $2 ; d=20 \mathrm{~m}$.

the first, denoted by $U n c$, is the ratio $E_{i g}$ for the unconstrained case; the entry denoted by $\left(\overline{\nabla E_{i g}}\right)_{\bar{U}}$ is the average gradient of $E_{i g}$ in the direction of the amplitude constraints, while $\left(\overline{\nabla E_{i g}}\right)_{\bar{F}}$ is the average gradient in the direction of the force constraint. Tables 2 and 3 confirm that the relative performance of the IC is more sensitive to the force constraint than the oscillation amplitude constraint, and that by reducing the maximum allowed PTO force the ratio $E_{i g} \rightarrow 1$. However, there is an exception for the case of the smallest peak period $\left(T_{p}=6 s\right)$ where a restriction of the maximum PTO force causes, on average, a degradation of the relative performance of the IC. In particular, by looking at Table 2, which lists the simulation results for the array layout 2 composed of WECs of geometry 2 , when $T_{\mathrm{p}}=6 \mathrm{~s}$ the sensitivity of $E_{i g}$ decreases in magnitude when the heading angle $\beta$ increases, which means that the performance degradation of the IC is reduced when the array is in terminator position $\left(\beta=90^{\circ}\right)$. When $T_{\mathrm{p}}=6 \mathrm{~s}$, the negative values of the gradient $\left(\overline{\nabla E_{i g}}\right)_{\bar{F}}$ for both layouts 2 and 3 (Tables 2 and 3, respectively) could be due the fact that the corresponding peak wavelength is approximately $56 \mathrm{~m}$ (in infinite depth water), which is slightly less than three times the inter-body distance $d=20 \mathrm{~m}$. Thus, the small ratio between the wavelength and $d$ may cause strong inter-body interference which penalise the IC.

In all cases, the magnitude of $\left(\overline{\nabla E_{i g}}\right)_{\bar{F}}$ is larger than the magnitude of $\left(\overline{\nabla E_{i g}}\right)_{\bar{U}}$; that is, $E_{i g}$ is more sensitive to variations in the force constraint than variations in the amplitude constraint, independently whether the variation is positive or negative.

The effect of the inter-body distance on $E_{i g}$, in conjunction with the wave heading angle and for the three layouts, is shown in Figs. 6-8. Each plot contains four curves, corresponding to different constraints configurations: the curve labelled "u" depicts $E_{i g}$ as function of the inter-body distance for the unconstrained case; the curves labelled "a" and "f" correspond the cases where only one type 

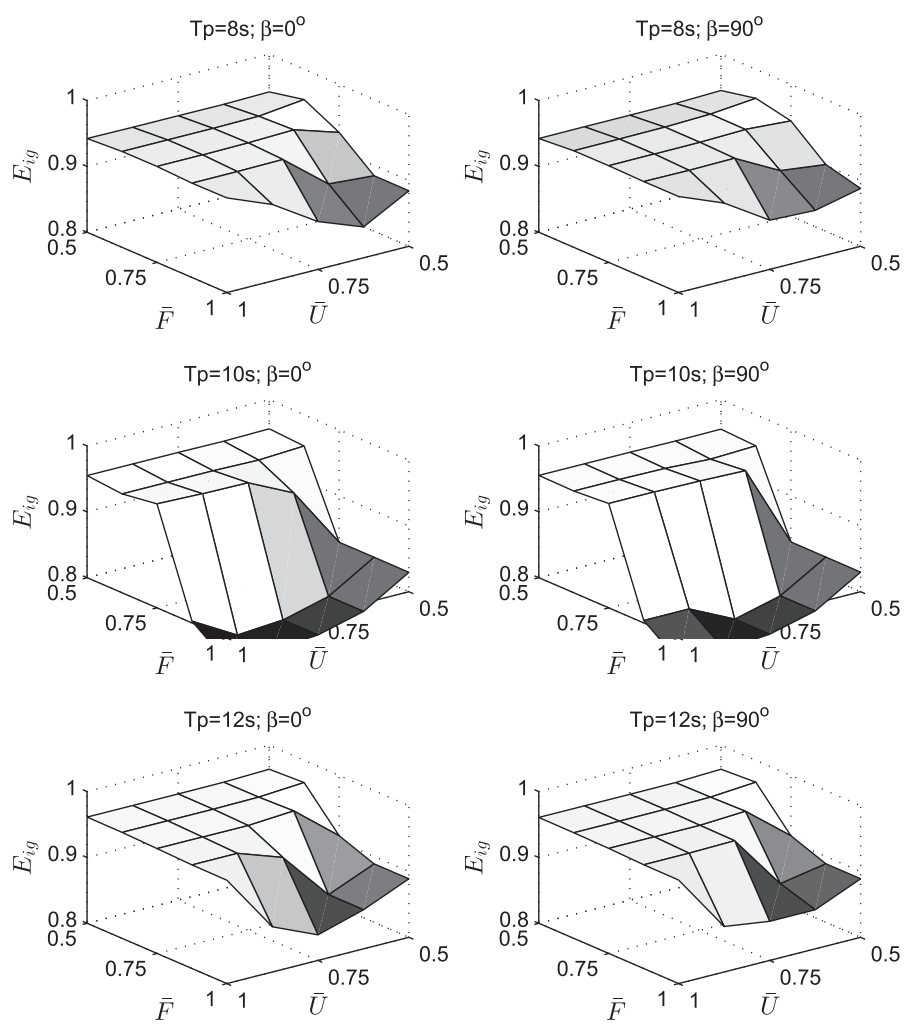

Fig. 5. Layout 3; geometry $2 ; d=20 \mathrm{~m}$.

Table 2

Layout 2; geometry 2; $d=20 \mathrm{~m}$.

\begin{tabular}{|c|c|c|c|c|c|}
\hline & & $T_{\mathrm{p}}=6 \mathrm{~s}$ & $T_{\mathrm{p}}=8 \mathrm{~s}$ & $T_{\mathrm{p}}=10 \mathrm{~s}$ & $T_{\mathrm{p}}=12 \mathrm{~s}$ \\
\hline \multirow[t]{3}{*}{$\beta=0^{\circ}$} & Unc & 0.953 & 0.971 & 0.880 & 0.975 \\
\hline & $\left(\overline{\nabla E_{i g}}\right)_{\bar{U}}$ & 0.007 & -0.015 & -0.002 & -0.032 \\
\hline & $\left(\overline{\nabla E_{i g}}\right)_{F}$ & -0.050 & -0.005 & 0.165 & 0.068 \\
\hline \multirow[t]{3}{*}{$\beta=30^{\circ}$} & Unc & 0.963 & 0.971 & 0.867 & 0.974 \\
\hline & $\left(\overline{\nabla E_{i g}}\right)_{\bar{U}}$ & 0.004 & -0.014 & 0.002 & -0.039 \\
\hline & $\left(\overline{\nabla E_{i g}}\right)_{\bar{F}}$ & -0.036 & 0.027 & 0.184 & 0.087 \\
\hline \multirow[t]{3}{*}{$\beta=45^{\circ}$} & Unc & 0.962 & 0.954 & 0.852 & 0.972 \\
\hline & $\left(\overline{\nabla E_{i g}}\right)_{U}$ & 0.008 & -0.008 & -0.005 & -0.041 \\
\hline & $\left(\overline{\nabla E_{i g}}\right)_{\bar{F}}$ & -0.021 & 0.066 & 0.209 & 0.097 \\
\hline \multirow[t]{3}{*}{$\beta=60^{\circ}$} & Unc & 0.957 & 0.914 & 0.834 & 0.919 \\
\hline & $\left(\overline{\nabla E_{i g}}\right)_{U}$ & 0.010 & 0.011 & 0.029 & -0.028 \\
\hline & $\left(\overline{\nabla E_{i g}}\right)_{F}$ & -0.011 & 0.099 & 0.235 & 0.145 \\
\hline \multirow[t]{3}{*}{$\beta=90^{\circ}$} & Unc & 0.945 & 0.897 & 0.813 & 0.938 \\
\hline & $\left(\overline{\nabla E_{i g}}\right)_{\bar{U}}$ & 0.014 & 0.017 & 0.008 & -0.041 \\
\hline & $\left(\overline{\nabla E_{i g}}\right)_{\bar{F}}$ & -0.016 & 0.111 & 0.260 & 0.151 \\
\hline
\end{tabular}


Table 3

Layout 3; geometry 2; $d=20 \mathrm{~m}$.

\begin{tabular}{|c|c|c|c|c|c|}
\hline & & $T_{\mathrm{p}}=6 \mathrm{~s}$ & $T_{\mathrm{p}}=8 \mathrm{~s}$ & $T_{\mathrm{p}}=10 \mathrm{~s}$ & $T_{\mathrm{p}}=12 \mathrm{~s}$ \\
\hline \multirow{3}{*}{$\beta=0^{\circ}$} & Unc & 0.930 & 0.944 & 0.764 & 0.957 \\
\hline & $\left(\overline{\nabla E_{i g}}\right)_{U}$ & 0.005 & -0.051 & -0.013 & -0.072 \\
\hline & $\left(\overline{\nabla E_{i g}}\right)_{\bar{F}}$ & -0.015 & 0.098 & 0.344 & 0.158 \\
\hline \multirow{3}{*}{$\beta=30^{\circ}$} & Unc & 0.933 & 0.942 & 0.763 & 0.957 \\
\hline & $\left(\overline{\nabla E_{i g}}\right)_{\bar{U}}$ & -0.005 & -0.054 & 0.017 & -0.071 \\
\hline & $\left(\bar{\nabla} E_{i g}\right)_{\bar{F}}$ & -0.022 & 0.133 & 0.345 & 0.160 \\
\hline \multirow[t]{3}{*}{$\beta=45^{\circ}$} & Unc & 0.924 & 0.942 & 0.763 & 0.957 \\
\hline & $\left(\overline{\nabla E_{i g}}\right)_{\bar{U}}$ & 0.000 & -0.047 & -0.014 & -0.072 \\
\hline & $\left(\overline{\nabla E_{i g}}\right)_{F}$ & -0.015 & 0.110 & 0.345 & 0.159 \\
\hline \multirow[t]{3}{*}{$\beta=60^{\circ}$} & Unc & 0.922 & 0.944 & 0.764 & 0.957 \\
\hline & $\left(\overline{\nabla E_{i g}}\right)_{U}$ & 0.009 & -0.050 & -0.015 & -0.071 \\
\hline & $\left(\overline{\nabla E_{i g}}\right)_{\bar{F}}$ & -0.014 & 0.109 & 0.344 & 0.158 \\
\hline \multirow[t]{3}{*}{$\beta=90^{\circ}$} & Unc & 0.927 & 0.946 & 0.765 & 0.957 \\
\hline & $\left(\overline{\nabla E_{i g}}\right)_{0}$ & 0.011 & -0.037 & -0.016 & -0.071 \\
\hline & $\left(\overline{\nabla E_{i g}}\right)_{\bar{F}}$ & -0.025 & 0.087 & 0.343 & 0.149 \\
\hline
\end{tabular}
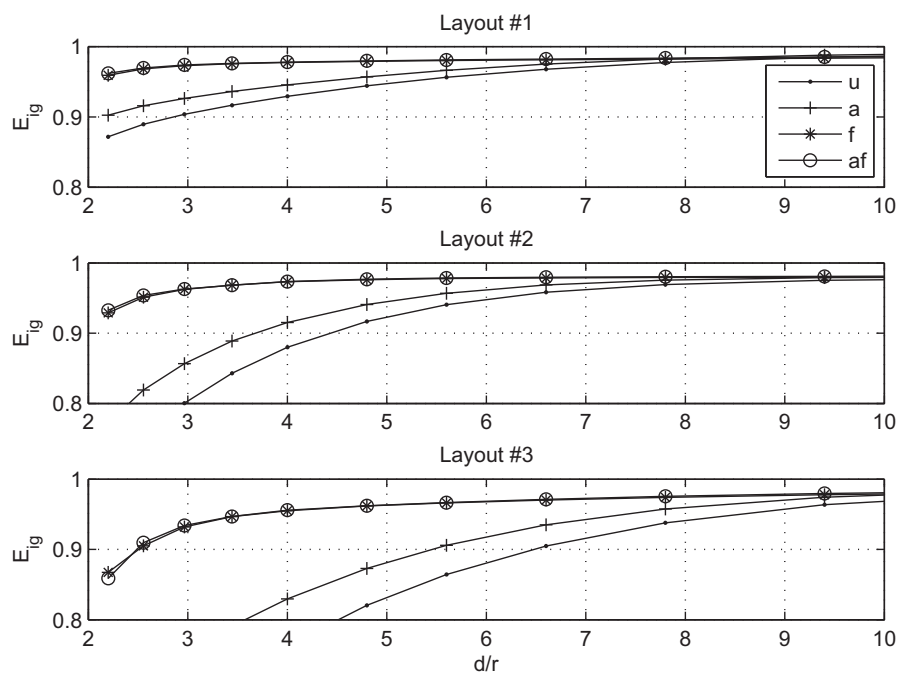

Fig. 6. Geometry $2 ; T_{p}=10 \mathrm{~s} ; \beta=0^{\circ}$.

of constraint is active and it is set to its minimum value considered in this paper, the normalized value of which is 0.5 . More in detail, the curve labelled as "a" is obtained by imposing $\bar{U}=0.5$ and leaving the PTO force unconstrained, while the curve " $\mathrm{f}$ " is obtained by setting $\bar{F}=0.5$. The last curve ("af") is obtained by simulating the system when both the normalized PTO force and normalized oscillation amplitude constraints are set to 0.5 .

As expected, the IC behaves similarly to the GC in terms of total absorbed energy (the ratio $E_{i g} \rightarrow 1$ ) for large inter-body spacing. In fact, the inter-body hydrodynamic interactions (both radiation and diffraction) decrease with increasing distance, and the error introduced in the control model used by the IC becomes negligible. 

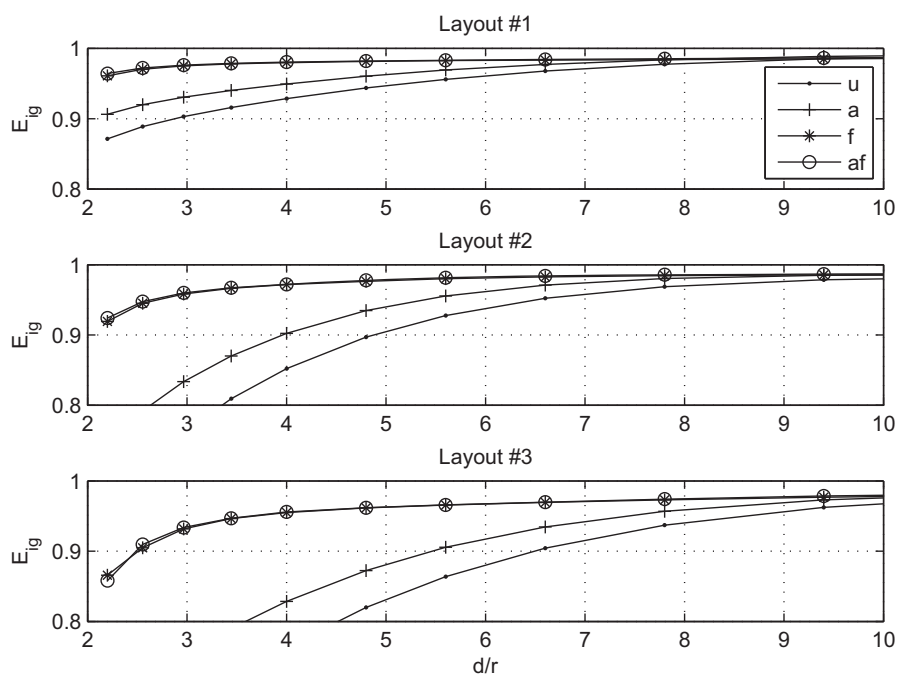

Fig. 7. Geometry $2 ; T_{p}=10 \mathrm{~s} ; \beta=45^{\circ}$.
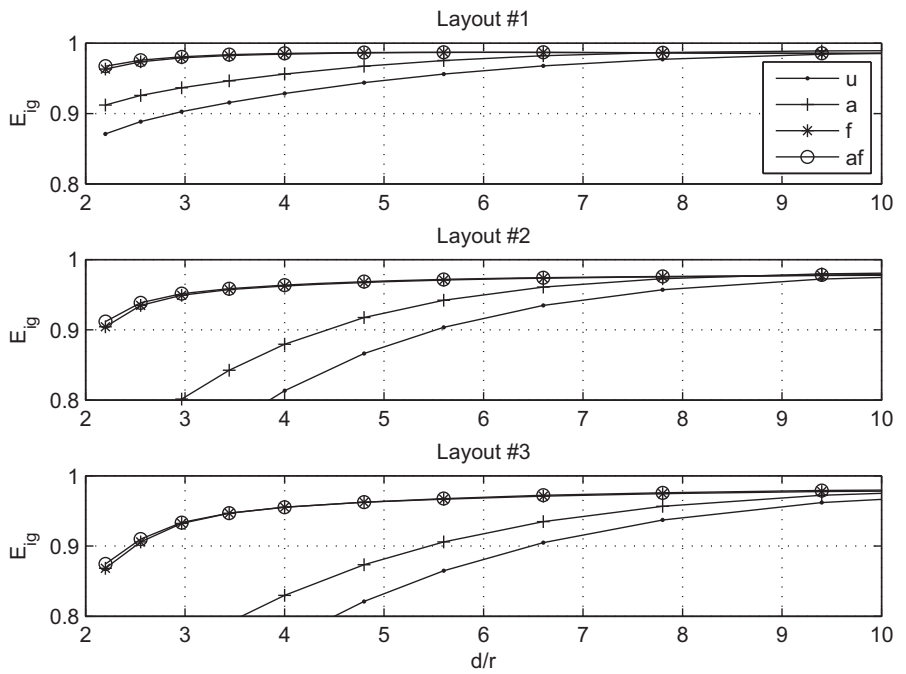

Fig. 8. Geometry $2 ; T_{p}=10 \mathrm{~s} ; \beta=90^{\circ}$.

Figs. 6-8 also highlight how restrictions in the motion and PTO force causes the performance of the IC to improve when compared to GC, because the curves referring to any constrained configuration ("a", "f", "af") are always above the curve of the unconstrained motion and force ("u"). It is also evident from Figs. 6-8 that the effect of the PTO force constraint on $E_{i g}$ is larger than the effect of the motion constraint, as the curves "f" and 'af" are always overlapped; this means that a variation on the amplitude constraint has no effect on $E_{i g}$ when the normalized force constraint is set to $\bar{F}=0.5$.

Figs. 6-8 correspond to simulations performed with the same geometry of the WEC ( $G 2$ ) and same sea state $\left(T_{p}=10 \mathrm{~s}\right)$, and to different wave heading angles $\beta$. By comparing these figures, it can be noted that $E_{\text {ig }}$ for layout 2 (linear array with three WECs) is the most sensitive to $\beta$, while for the layout 1 , which is composed of two devices, is less sensitive; when considering layout $3, E_{i g}$ is practically unaffected by the wave heading angle. 
Table 4

$d=20 \mathrm{~m} ; T_{p}=8 \mathrm{~s}$; geometry 2 .

\begin{tabular}{|c|c|c|c|c|}
\hline & & L 1 & L 2 & L 3 \\
\hline \multirow[t]{3}{*}{$\beta=0^{\circ}$} & Unc & 0.976 & 0.971 & 0.944 \\
\hline & $\left(\overline{\nabla E_{i g}}\right)_{U}$ & -0.009 & -0.015 & -0.051 \\
\hline & $\left(\overline{\nabla E_{i g}}\right)_{\bar{F}}$ & 0.018 & -0.005 & 0.098 \\
\hline \multirow{3}{*}{$\beta=30^{\circ}$} & Unc & 0.977 & 0.971 & 0.942 \\
\hline & $\left(\overline{\nabla E_{i g}}\right)_{\bar{U}}$ & -0.011 & -0.014 & -0.054 \\
\hline & $\left(\overline{\nabla E_{i g}}\right)_{\bar{F}}$ & 0.022 & 0.027 & 0.133 \\
\hline \multirow{3}{*}{$\beta=45^{\circ}$} & Unc & 0.977 & 0.954 & 0.942 \\
\hline & $\left(\overline{\nabla E_{i g}}\right)_{\bar{U}}$ & -0.014 & -0.008 & -0.047 \\
\hline & $\left(\overline{\nabla E_{i g}}\right)_{F}$ & 0.023 & 0.066 & 0.110 \\
\hline \multirow[t]{3}{*}{$\beta=60^{\circ}$} & Unc & 0.969 & 0.914 & 0.944 \\
\hline & $\left(\overline{\nabla E_{i g}}\right)_{\bar{U}}$ & -0.006 & 0.011 & -0.050 \\
\hline & $\left(\overline{\nabla E_{i g}}\right)_{F}$ & 0.032 & 0.099 & 0.109 \\
\hline \multirow[t]{3}{*}{$\beta=90^{\circ}$} & Unc & 0.953 & 0.897 & 0.946 \\
\hline & $\left(\overline{\nabla E_{i g}}\right)_{U}$ & 0.005 & 0.017 & -0.037 \\
\hline & $\left(\overline{\nabla E_{i g}}\right)_{\bar{F}}$ & 0.048 & 0.111 & 0.087 \\
\hline
\end{tabular}

Table 5

$d=20 \mathrm{~m} ; T_{p}=10 \mathrm{~s}$; geometry 2 .

\begin{tabular}{|c|c|c|c|c|}
\hline & & L 1 & L 2 & L 3 \\
\hline \multirow{3}{*}{$\beta=0^{\circ}$} & Unc & 0.929 & 0.880 & 0.764 \\
\hline & $\left(\overline{\nabla E_{i g}}\right)_{U}$ & -0.005 & -0.002 & -0.013 \\
\hline & $\left(\overline{\nabla E_{i g}}\right)_{\bar{F}}$ & 0.087 & 0.165 & 0.344 \\
\hline \multirow[t]{3}{*}{$\beta=0^{\circ}$} & Unc & 0.929 & 0.867 & 0.763 \\
\hline & $\left(\overline{\nabla E_{i g}}\right)_{\bar{U}}$ & -0.003 & 0.002 & 0.017 \\
\hline & $\left(\overline{\nabla E_{i g}}\right)_{\bar{F}}$ & 0.088 & 0.184 & 0.345 \\
\hline \multirow[t]{3}{*}{$\beta=0^{\circ}$} & Unc & 0.929 & 0.852 & 0.763 \\
\hline & $\left(\overline{\nabla E_{i g}}\right)_{\bar{U}}$ & 0.004 & -0.005 & -0.014 \\
\hline & $\left(\overline{\nabla E_{i g}}\right)_{F}$ & 0.090 & 0.209 & 0.345 \\
\hline \multirow[t]{3}{*}{$\beta=0^{\circ}$} & Unc & 0.928 & 0.834 & 0.764 \\
\hline & $\left(\overline{\nabla E_{i g}}\right)_{\bar{U}}$ & 0.008 & 0.029 & -0.015 \\
\hline & $\left(\overline{\nabla E_{i g}}\right)_{F}$ & 0.092 & 0.235 & 0.344 \\
\hline \multirow[t]{3}{*}{$\beta=0^{\circ}$} & Unc & 0.928 & 0.813 & 0.765 \\
\hline & $\left(\overline{\nabla E_{i g}}\right)_{U}$ & 0.007 & 0.008 & -0.016 \\
\hline & $\left(\overline{\nabla E_{i g}}\right)_{\bar{F}}$ & 0.096 & 0.260 & 0.343 \\
\hline
\end{tabular}

The behaviour of $E_{i g}$ with respect to the heading angle and the array layout is confirmed by looking and the data in Tables 4 and 5, which list the values of $E_{i g}$ and its gradients for additional values of $\beta$ and for two sea states ( $T_{p}=8 \mathrm{~s}$ for Table 4 and $T_{p}=10 \mathrm{~s}$ for Table 5 ).

The effect of the WEC geometry on the relative performance of IC compared to GC can be inferred form data in Tables 6 and 7, which list the values of $E_{i g},\left(\overline{\nabla E_{i g}}\right)_{\bar{U}}$ and $\left(\overline{\nabla E_{i g}}\right)_{\bar{F}}$ for the three layouts and the three WEC geometries; both tables refer to the same wave heading angle $\beta=90^{\circ}$ and normalized distance $d / r=4$. The IC performs worse for the device with the strongest radiative properties $(G=3)$ in all the situations; this observation was expected, because a stronger interaction implies a larger effect of the error in the control model used by the IC, with the consequent degradation in relative performance. 
Table 6

$T_{p}=8 s ; d / r=4 ; \beta=90^{\circ}$.

\begin{tabular}{lllll}
\hline & & L 1 & L 2 & L 3 \\
\hline G 1 & Unc & 0.966 & 0.925 & 0.975 \\
& $\left(\overline{\nabla E_{i g}}\right)_{\bar{U}}$ & 0.005 & 0.009 & -0.015 \\
& $\left(\overline{\nabla E_{i g}}\right)_{\bar{F}}$ & 0.017 & 0.083 & 0.015 \\
G 2 & Unc & 0.953 & 0.897 & 0.946 \\
& $\left(\overline{\nabla E_{i g}}\right)_{\bar{U}}$ & 0.005 & 0.017 & -0.037 \\
G 3 & $\left(\overline{\nabla E_{i g}}\right)_{\bar{F}}$ & 0.048 & 0.111 & 0.087 \\
& Unc & 0.924 & 0.868 & 0.917 \\
& $\left(\overline{\nabla E_{i g}}\right)_{\bar{U}}$ & 0.025 & 0.031 & -0.030 \\
& $\left(\overline{\nabla E_{i g}}\right)_{\bar{F}}$ & 0.063 & 0.113 & 0.119 \\
\hline
\end{tabular}

Table 7

$T_{p}=10 \mathrm{~s} ; d / r=4 ; \beta=90^{\circ}$.

\begin{tabular}{lllll}
\hline & & L 1 & L 2 & L 3 \\
\hline G 1 & Unc & 0.965 & 0.896 & 0.875 \\
& $\left(\overline{\nabla E_{i g}}\right)_{\bar{U}}$ & 0.009 & 0.021 & 0.006 \\
& $\left(\overline{\nabla E_{i g}}\right)_{\bar{F}}$ & 0.037 & 0.145 & 0.175 \\
G 2 & Unc & 0.928 & 0.813 & 0.765 \\
& $\left(\overline{\nabla E_{i g}}\right)_{U}$ & 0.007 & 0.008 & -0.016 \\
G 3 & $\left(\overline{\nabla E_{i g}}\right)_{\bar{F}}$ & 0.096 & 0.260 & 0.343 \\
& Unc & 0.897 & 0.761 & 0.690 \\
& $\left(\overline{\nabla E_{i g}}\right)_{U}$ & 0.021 & 0.033 & -0.041 \\
& $\left(\overline{\nabla E_{i g}}\right)_{\bar{F}}$ & 0.139 & 0.307 & 0.439 \\
\hline
\end{tabular}

\section{Conclusions}

The results in Section 4 clearly indicate that a significant performance improvement (up to $10 \%$, or more) can be obtained using global control of arrays, compared to independent control. Moreover, the methodology applied in this paper provides an upper bound for relative the performance of the Independent Controller when compared to the Global Controller. Thus, considering the more realistic case where the ICs will not have time to fully converge on a quasi steady-state (which assumes that the sea state is strictly stationary), significantly greater benefits of GC are indicated, with improvements of greater than $20 \%$ possible for small $d$. The performance of both GC and IC will be affected also by the prediction of wave elevation or excitation force, which has not been studied in this paper because the authors have applied the separation principle (Section 3 ).

The benefits of GC are greatest for layouts which have maximum device interaction (e.g. layout 3 in our analysis) and devices which have strong radiation properties (e.g. geometry 3 , which has a large radius/draught ratio). The degree of benefit of the GC over the ICs, as expected, diminishes as the interaction between devices decreases. Therefore, the greatest benefit of GC is likely to be manifest for closely-packed arrays of WECs, such as Wavestar [4], Fred Olsen Lifesaver [5] , Manchester bobber [6] and Trident Technologies [7] systems.

In addition, the effect of PTO force and oscillation amplitude constraints on the relative performance of the IC with respect to the GC has been studied. The results show that by restricting the motion of the devices, or restricting the maximum force exerted by the PTO, generally reduces the performance gap between the IC and the GC. However, a significant reduction in the performance gap is only obtained for a restriction in the maximum allowed PTO force, while a restriction on the device motion affects the ratio $E_{i g}$ with a smaller magnitude. Also, it has to be noted that the 
performance gap may not be significantly reduced in practice because the total absorbed energy for the IC is an upper bound, which can only be obtained in an ideal, non realistic situation.

\section{Acknowledgment}

The authors gratefully acknowledge the financial support of Enterprise Ireland under contract TD/ 2009/0331.

\section{References}

[1] M. Carcas, The Pelamis Wave Energy Converter, vol. 10, Ocean Power Delivery Ltd, 2010.

[2] T. Whittaker, M. Folley, Nearshore oscillating wave surge converters and the development of oyster, Philosophical Transactions of the Royal Society A: Mathematical, Physical and Engineering Sciences 370 (1959) (2012) 345-364. Available from http://rsta.royalsocietypublishing.org/content/370/1959/345.abstract (Online).

[3] M. Draper, More than just a ripple: ocean power technologies sets its sights high, Refocus 7 (1) (2006) 54-56. Available from http://www.sciencedirect.com/science/article/pii/S1471084606705200 (online).

[4] R.H. Hansen, M.M. Kramer, Modelling and control of the wavestar prototype, in 9th European Wave and Tidal Energy Conference, EWTEC, 2011.

[5] C.M. Sandvik, Wave-to-wire model of the wave energy converter bolt2: control and power extraction with an all-electric power take-off system, Ph.D. dissertation, Norwegian University of Science and Technology, 2012.

[6] X. Garnaud, C.C. Mei, Wave-power extraction by a compact array of buoys, Journal of Fluid Mechanics 635 (2009) 389-413.

[7] R. Antonutti, G.E. Hearn, Optimisation of point-absorber arrays, in: 9th European Wave and Tidal Energy Conference, EWTEC, 2011.

[8] X. Garnaud, C. Mei, Comparison of wave power extraction by a compact array of small buoys and by a large buoy, Renewable Power Generation IET 4 (6) (2010) 519-530.

[9] D. Evans, Some theoretical aspects of three-dimensional wave-energy absorbers, in: First Symposium on Wave Energy Utilization, Gothenburg. Chalmers University of Technology, 1979.

[10] J. Falnes, Radiation impedance matrix and optimum power absorption for interacting oscillators in surface waves, Applied Ocean Research 2 (2) (1980) 75-80. Available from http://www.sciencedirect.com/science/article/B6V1V-47YVV8H-4/2/ 65fc8b0ba1fd466dcee3c663f11b86f4 (online).

[11] P.A. Justino, A. Clément, Hydrodynamic performance for small arrays of submerged spheres, in: 5th European Wave and Tidal Energy Conference, EWTEC, 2003, pp. 266-273.

[12] B. Child, V. Venugopal, Non-optimal tuning of wave energy device arrays, in 2nd International Conference on Ocean Energy, ICOE, 2008.

[13] S. Bellew, T. Stallard, P. Stansby, Optimisation of a heterogeneous array of heaving bodies, in: 8th European Wave and Tidal Energy Conference, EWTEC, 2009.

[14] G. De Backer, M. Vantorre, C. Beels, J. De Rouck, P. Frigaard, Power absorption by closely spaced point absorbers in constrained conditions, Renewable Power Generation, IET 4 (6) (2010) 579-591.

[15] P. Ricci, J.-B. Saulnier, A.F. de O. Falcão, Point-absorber arrays: a configuration study off the Portuguese west-coast, in: 7th European Wave and Tidal Energy Conference, EWTEC, 2007.

[16] M. Folley, T. Whittaker, The effect of sub-optimal control and the spectral wave climate on the performance of wave energy converter arrays, Applied Ocean Research 31 (4) (2009) 260-266. Available from http://www.sciencedirect.com/science/ article/pii/S0141118709000947 (online).

[17] J. Cruz, R. Sykes, P. Siddorn, R. Taylor, Estimating the loads and energy yield of arrays of wave energy converters under realistic seas, Renewable Power Generation, IET 4 (6) (2010) 488-497.

[18] G.P. Thomas, D.V. Evans, Arrays of three-dimensional wave-energy absorbers, Journal of Fluid Mechanics 108 (1981) $67-$ 88.

[19] D. Evans, Maximum wave-power absorption under motion constraints, Applied Ocean Research 3 (4) (1981) $200-203$. Available from http://www.sciencedirect.com/science/article/B6V1V-4816TBH-24/2/b81da13cab0165d97e4d81d93de23c 41 (online).

[20] J. Falnes, K. Budal, Wave-power absorption by parallel rows of interacting oscillating bodies, Applied Ocean Research 4 (4) (1982) 194-207. Available from http://www.sciencedirect.com/science/article/pii/S0141118782800266 (online).

[21] C. Fitzgerald, G. P. Thomas, A preliminary study of the optimal formation of an array of wave power devices, in: 7 th European Wave and Tidal Energy Conference, EWTEC, 2007.

[22] J. Hals, J. Falnes, T. Moan, Constrained optimal control of a heaving buoy wave-energy converter, Journal of Offshore Mechanics and Arctic Engineering 133 (1) (2011) 011401. Available from http://link.aip.org/link/?JOM/133/011401/1 (online).

[23] J.A. M. Cretel, G. Lightbody, G.P. Thomas, A.W. Lewis, Maximisation of energy capture by a wave-energy point absorber using model predictive control, in: IFAC World Congress, 2011.

[24] J. Westphalen, G. Bacelli, P. Balitsky, J.V. Ringwood, Control strategies for arrays of wave energy devices, in: 9th European Wave and Tidal Energy Conference, EWTEC, 2011.

[25] W. Cummins, The impulse response function and ship motions, David Taylor Model Basin DTNSRDC, Tech. Rep., 1962.

[26] T. Ogilvie, Recent progress toward the understanding and prediction of ship motions, in: Proc. Fifth Symposium on Naval Hydrodynamics, Bergen, Norway, 1964.

[27] T. Perez, T.I. Fossen, Time- vs. frequency-domain identification of parametric radiation force models for marine structures at zero speed, Modeling, Identification and Control 29 (1) (2008) 1-19.

[28] WAMIT Inc, www.wamit.com. Available from http://www.wamit.com (online). 
[29] J.F. Douglas, J. Gasiorek, J. Swaffield, Fluid Mechanics, fifth ed., Pearson/Prentice Hall, 2005.

[30] S. Bellew, T. Stallard, Linear modelling of wave device arrays and comparison to experimental and second order models, in: International Workshop for Water Waves and Floating Bodies, 2010, pp. 2-5. Available from http://www.iwwwfb.org/ Abstracts/iwwwfb25/iwwwfb25_03.pdf (online).

[31] M.A. Bhinder, A. Babarit, L. Gentaz, P. Ferrant, Assessment of viscous damping via 3D-cfd modelling of a floating wave energy device, in: 9th European Wave and Tidal Energy Conference, EWTEC, 2011.

[32] G.M. Terra, W.J. van de Berg, L.R.M. Maas, Experimental verification of lorentz' linearization procedure for quadratic friction, Fluid Dynamics Research 36 (1995) 175-188.

[33] M. Folley, T. Whittaker, J. van't Hoff, The design of small seabed-mounted bottom-hinged wave energy converters, in: 7th European Wave and Tidal Energy Conference, EWTEC, 2007.

[34] F. Fusco, J. Ringwood, A study of the prediction requirements in real-time control of wave energy converters, IEEE Transactions on Sustainable Energy 3 (1) (2012) 176-184.

[35] G. Bacelli, J.V. Ringwood, J.-C. Gilloteaux, A control system for a self-reacting point absorber wave energy converter subject to constraints, in: IFAC World Congress, 2011.

[36] H. Kwakernaak, R. Sivan, Linear Optimal Control Systems, vol. 172, Wiley-Interscience, New York, 1972.

[37] J. Nocedal, S.J. Wright, Numerical Optimization, 2nd ed., Springer, New York, 2006. 